or Fisher's exact tests as appropriate. Multivariate analysis including logistic regression models will be used to test for associations between maternal characteristics and SP resistance. Level of significance will be set at $\mathrm{p}<0.05$.

Conclusion In a malaria-endemic country like Nigeria with a large at-risk population, information on the effectiveness of chemoprevention is essential. Determining the proportion and extent of relevant molecular markers within the population offers an invaluable tool for epidemiological surveillance of SP resistance within this endemic setting.

\section{PO 8503 EPIDEMIOLOGY, CO-INFECTIONS AND HAEMATOLOGICAL FEATURES OF SCHISTOSOMIASIS IN SCHOOL-AGED CHILDREN LIVING IN LAMBARÉNÉ, GABON}

${ }^{1} J$ ean Claude Dejon Agobe*, 'Yabo J Honkpehedij, 'Jeannot Fréjus Zinsou, 'JeanRonald Edoa, 'Bayodé R Adegbite, 'Mohamed Duali, ${ }^{1}$ Fabrice L Mougeni, 'Bertrand Lell, ${ }^{2}$ Peter G Kremsner, ${ }^{3}$ Martin P Grobusch, ${ }^{1}$ Ayola Akim Adegnika. ${ }^{1}$ Centre de Recherches Médicales de Lambaréné, Libreville, Gabon; 'Institut für Tropenmedizin, Eberhard Karls Universität Tübingen and German Center for Infection Research (DZIF), Germany; ${ }^{3}$ Academic Medical Center, University of Amsterdam, the Netherlands

\subsection{6/bmjgh-2019-EDC.123}

Background Schistosomiasis is a highly prevalent parasitic infection in Central Africa, where co-endemicity with other parasitic infections is common, and schistosomiasis outcomes can be affected by those other infections. Therefore, proper schistosomiasis control needs epidemiological data accounting for co-infections, too. In this present study, our objective was to determine the epidemiological situation around schistosomiasis in Lambaréné.

Methods A cross-sectional study was conducted among schoolaged children living in Lambaréné. Urine filtration exam was performed for the detection of Schistosoma eggs. Kato-Katz and stool culture (Coproculture and Harada-Mori) techniques were used for the detection of soil-transmitted helminths. Detection of Plasmodium spp. and blood microfilariae was performed applying light microscopy. Risk factors for schistosomiasis and factors associated with schistosomiasis were investigated; haematology parameters evaluated.

Results A total of 614 school children with available schistosomiasis status were included in the analysis. Mean age was $10.9(\mathrm{SD}=2.7)$ years, with a 0.95 boy-to-girl sex ratio. The prevalence of schistosomiasis was $26 \%$. No risk factors except human-water contact were associated with schistosomiasis. Only Trichuris trichiura co-infection was associated with an increased odd $(\mathrm{aOR}=2.3, p$-value $=0.048)$ to be infected with schistosomiasis. Full blood counts showed a decrease of haemoglobin level and increase of WBC and platelet levels among the schistosoma-infected children. Haematuria was found associated with schistosomiasis $(\mathrm{aOR}=14.5, p$-value $<0.001)$ and was suitable to predict the disease.

Conclusion The prevalence of schistosomiasis is moderate in Lambaréné where human-water contact remains the main risk factor and praziquantel is available for treatment. Trichuriasis is associated with increased risk to be infected. Children with schistosomiasis exhibit a distinct full blood count profile and haematuria is found to be more suitable to predict infection. However, it is desirable to implement comprehensive approaches beyond chemotherapy for schistosomiasis control in this area as recommended by WHO.

\section{PO 8504 EFFECT OF INCREASED USER FEES IN ACCESSING NEW TUBERCULOSIS DIAGNOSTIC SERVICES IN TANZANIA}

${ }^{1}$ Esther Ngadaya*, 'Godfather Kimaro, ${ }^{1}$ Ramadhani Shemtandulo, ${ }^{1}$ Erica Sandi, ${ }^{2}$ Sunday Simsokwe, ${ }^{2}$ Oliva Nguma, ${ }^{3}$ Omari Kibwana, ${ }^{4}$ Nicholaus Mgina, ${ }^{1}$ Godfrey S Mfinanga. 'National Institute for Medical Research, Muhimbili Research Centre, Dar es Salaam, Tanzania; ${ }^{2}$ Sumbawanga Regional Hospital, Sumbawanga, Tanzania; ${ }^{3}$ Mnazi Mmoja Hospital Zanziba, Tanzania; ${ }^{4}$ Musoma Regional Hospital, Tanzania

\subsection{6/bmjgh-2019-EDC.124}

Background While user fees in healthcare systems have been associated with quality improvement, a substantial increase may have a detrimental effect. This paper reports on the effects of increasing user fees on utilisation of TB diagnostic services in Tanzania.

Methods We retrospectively analysed data on TB diagnostic services utilisation between July 2013 and June 2015 in Mnazi Mmoja Zanzibar (MMZ), Musoma and Sumbawanga hospitals. In July 2014, user fees in Musoma were increased substantially from 2 to 5 US dollar; Sumbawanga increased the fees stepwise, from 1 to 2 US dollar in July 2014, and from 2 to 3 US dollar in January 2015 MMZ did not raise the fees. We compared TB services utilisation before and after introduction of user fees.

Results Out of 7483 presumptive TB patients registered in all sites, 50.2\% were males. Over half (3969) were registered before the user fee was increased. Among 3969, 1579 (39.8\%) were from Musoma, 922 (23.2\%) from Sumbawanga and 1468 (37.0\%) from MMZ. Of the 3514 patients registered after the introduction of user fees, 983 (28\%), 952 (27.1\%) and 1579 (44.9\%) patients were from Musoma, Sumbawanga and MMZ, respectively. The number of presumptive TB patients seeking TB diagnostic services at Musoma decreased significantly by $38 \%$ from 1579 to 983 after the increase of user fees $(p=0.001)$. More females $(817 ; 51.8 \%$ vs 458, 35.9\%) attended Musoma before user fees were increased as compared to males whose attendance did not differ much $(761 ; 48.2 \%$ vs $525 ; 53.4 \%) ;(p=0.01)$. There was no significant decrease of patients at Sumbawanga and MMZ. Conclusion There was a significant decrease in the number of presumptive TB patients who accessed new TB diagnostic services in Musoma after a substantial increase of user fees, the effect was stronger among women. Although user fees are beneficial, they should be increased stepwise so as not to affect service utilisation.

\section{PO 8505 LEISHMANIASIS IN ANGOLA - AN EMERGING DISEASE?}

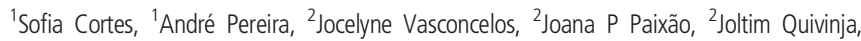
${ }^{2} J o a n a$ De Morais Afonso, 'José M Cristóvão, 'Lenea Campino. ' Global Health and Tropical Medicine Center, Instituto de Higiene e Medicina Tropical (IHMT), Universidade Nova de Lisboa, Portugal; ${ }^{2}$ Instituto Nacional de Saúde Pública, Ministério da Saúde de Angola, Angola

\subsection{6/bmjgh-2019-EDC.125}

Background Poverty, lack of resources, inadequate treatments and control programmes exacerbate the impact of infectious diseases in the developing world. Leishmaniasis is a vectorborne disease that is among the ten major neglected tropical diseases. Although endemic in more than 90 countries, the ones most affected, representing over $90 \%$ of new cases, are Bangladesh, Brazil, Ethiopia, India, Kenya, Nepal, and Sudan. In Africa south of the equator, the impact of leishmaniasis is 